\title{
Therapy-Related Toxicity
}

National Cancer Institute

\section{Source}

National Cancer Institute. Therapy-Related Toxicity. NCI Thesaurus. Code C42607.

The finding of bodily harm due to the poisonous effects associated with the action or administration of therapeutic agents. 